\title{
Surgical Management of a Large Periapical Lesion - A Two Year Follow Up
}

\author{
Aishwarya Das ${ }^{1}$, Iffat Nasim² \\ ${ }^{1}$ Department of Conservative Dentistry and Endodontics, Saveetha Dental College, \\ Chennai, Tamilnadu, India. ${ }^{2}$ Department of Conservative Dentistry and Endodontics \\ Saveetha Dental College, Chennai, Tamil Nadu, India.
}

INTRODUCTION

A successful endodontic therapy depends on resolution of all the clinical signs and symptoms with complete periapical repair or regeneration. Sometimes conventional root canal therapy fails because of which we have to resort to periapical surgery. ${ }^{1}$ Periapical surgery removes the pathological tissue and leads to healing of the periapical lesion.2,3 Periapical surgery accounts for $3 \%-10 \%$ in endodontic practice. $^{4}$

Platelet-Rich Fibrin (PRF) is a platelet concentrate with specific composition, three-dimensional architecture, and has characteristics with all the constituents of a blood sample to favour wound healing. ${ }^{5}$ PRF contains numerous growth factors such as platelet-derived growth factor (PDGF), transforming growth factor $\beta 1$ (TGF $\beta 1$ ), insulin-like growth factor (IGF) and exhibits numerous properties such as cell migration, cell attachment, cell proliferation, and differentiation ${ }^{6}$ facilitating the regenerative process of human body by utilizing the patient's own blood is a novel concept in dentistry. These blood clots initiate the process of healing and regeneration of the hard and soft tissues.7,8

$\mathrm{PRF}$ is like an interpositional biomaterial. It accelerates wound healing due to growth factor release. It is an easy and cost-effective way to obtain high concentrations of growth factors for tissue healing and regeneration. ${ }^{5}$

Any periapical lesion is a type of response of the bone around the apex of tooth that develops due to pulpal necrosis or extensive periodontal disease. Following endodontic treatment, repair or regeneration can occur depending on the microenvironmental causes. This case report exemplifies the use of platelet rich fibrin (PRF) and bone graft for the management of a large periapical lesion. Following endodontic treatment, periapical endodontic surgery was performed on a 41 year old male patient having a swelling in the buccal region of lower front teeth with a large defect evidenced radiographically. The surgical defect after curettage was filled with PRF, bone graft and guided tissue membrane and sutured. PRF along with bone graft accelerated the wound healing and induced bone formation.
Corresponding Author: Dr. Aishwarya Das. Mahalaxmi Flats, Besant Nagar, Chennai, Tamilnadu, India. E-mail: aishwarya.das@gmail.com

DOI: $10.14260 / \mathrm{jemds} / 2020 / 618$

How to Cite This Article: Das A, Nasim I. Surgical management of a large periapical lesion - a two year follow up. J Evolution Med Dent Sci 2020;9(38): 2839-2842, DOI:

$10.14260 /$ jemds/2020/618

Submission 14-06-2020,

Peer Review 11-08-2020,

Acceptance 19-08-2020,

Published 21-09-2020.

Copyright ( $(2020$ Aishwarya Das et al. This is an open access article distributed under Creative Commons Attribution License [Attribution 4.0 International (CC BY 4.0)] 


\section{PRESENTATION OF CASE}

A 41-year-old male patient reported to the outpatient department of our college complaining of a dull pain with a hard swelling on the outer surface of gum region in relation to lower front teeth for the past one month. History of trauma while playing cricket before eight years was revealed. On clinical examination, a swelling of $3 \mathrm{~cm} \times 1.5 \mathrm{~cm}$ size was seen over the buccal mucosa in relation to 33,34 and 35 [Figure 1].

Mild tenderness was felt on palpation over the apical mucosa in relation to 33,34 and 35 . These three teeth were not tender to percussion tests. Pulp sensitivity tests revealed that 33, 34 and 35 were non-vital. Preoperative digital radiograph showed presence of a large clearly demarcated lesion $(3 \mathrm{~cm} \times$ $2 \mathrm{~cm}$ in size) at the apex of 33, 34 and 35 [Figure 2]. Initially, root canal treatment was initiated in 33,34 and 35 and intracanal medicament of calcium hydroxide was given in all the three teeth. After one week, the calcium dressing was changed and repeated. There was no significant change in pain or swelling. Then periapical surgery in 33,34 and 35 was planned. Step back technique was used for root canal treatment till an apical size of \#50 in all the three teeth. The roots of these teeth were filled with gutta-percha using a suitable sealer employing the cold lateral condensation technique. Prior to the periapical surgery planned, the necessary routine blood investigations were done and were found to be within the normal range. Informed consent for the surgical procedure was obtained from the patient. Under local anaesthesia, an incision was given starting from the distal aspect of the lower left lateral incisor till the distal aspect of the lower left second. After the flap reflection, a perforated defect was observed with an absence of the labial cortical plate, measuring $3 \mathrm{~cm}, 2 \mathrm{~cm}$, and $2 \mathrm{~cm}$ in length, width, and depth respectively. Surgical enucleation was carefully carried out at the site of the lesion and the surgical area was copiously irrigated with betadine and saline solution [Figure 3].

The granulation tissue which was obtained was further transferred to the histopathological department for analysis. A long shank tapered fissure bur using a contra angle micro motor hand piece was used for the root end resection in teeth $33,34,35$. Root end cavity of $3 \mathrm{~mm}$ depth was prepared with diamond-coated ultrasonic surgical tip S12 90ND (Satelec / Acteon, Merignac, France). Mineral trioxide aggregate (MTA) was used as the retrograde filling material in all the three teeth [Figure 4]. To get the platelet concentrate, PRF, patient's own blood of around $15 \mathrm{ml}$ was used and centrifuged using REMI Centrifuge Machine for ten minutes under $3500 \mathrm{rpm}$. Bone graft material (Osseograft, Advanced Biotech Products, India) was taken and coupled with PRF and kept directly into the enucleated surgical site. A guided tissue regeneration membrane was placed as there was marginal bone loss present. Flap was stabilized followed by suturing using 3-0 black silk suture material (Sutures India Pvt. Ltd, Karnataka, India). Analgesics, antibiotics and $0.2 \%$ chlorhexidine mouthwash was prescribed to the patient for a week. Sutures were removed after one week later with satisfactory healing of the surgical site. Patient was reviewed at every three months [Figure 5] and any signs and symptoms of discomfort were assessed in these review appointments. Also radiographically and clinically the patient was examined. In the radiograph, bone regeneration was evident at the end of 24 months [Figure 6].

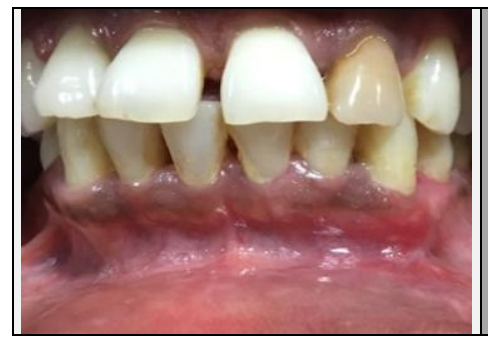

Figure 1.

Pre Op Clinical
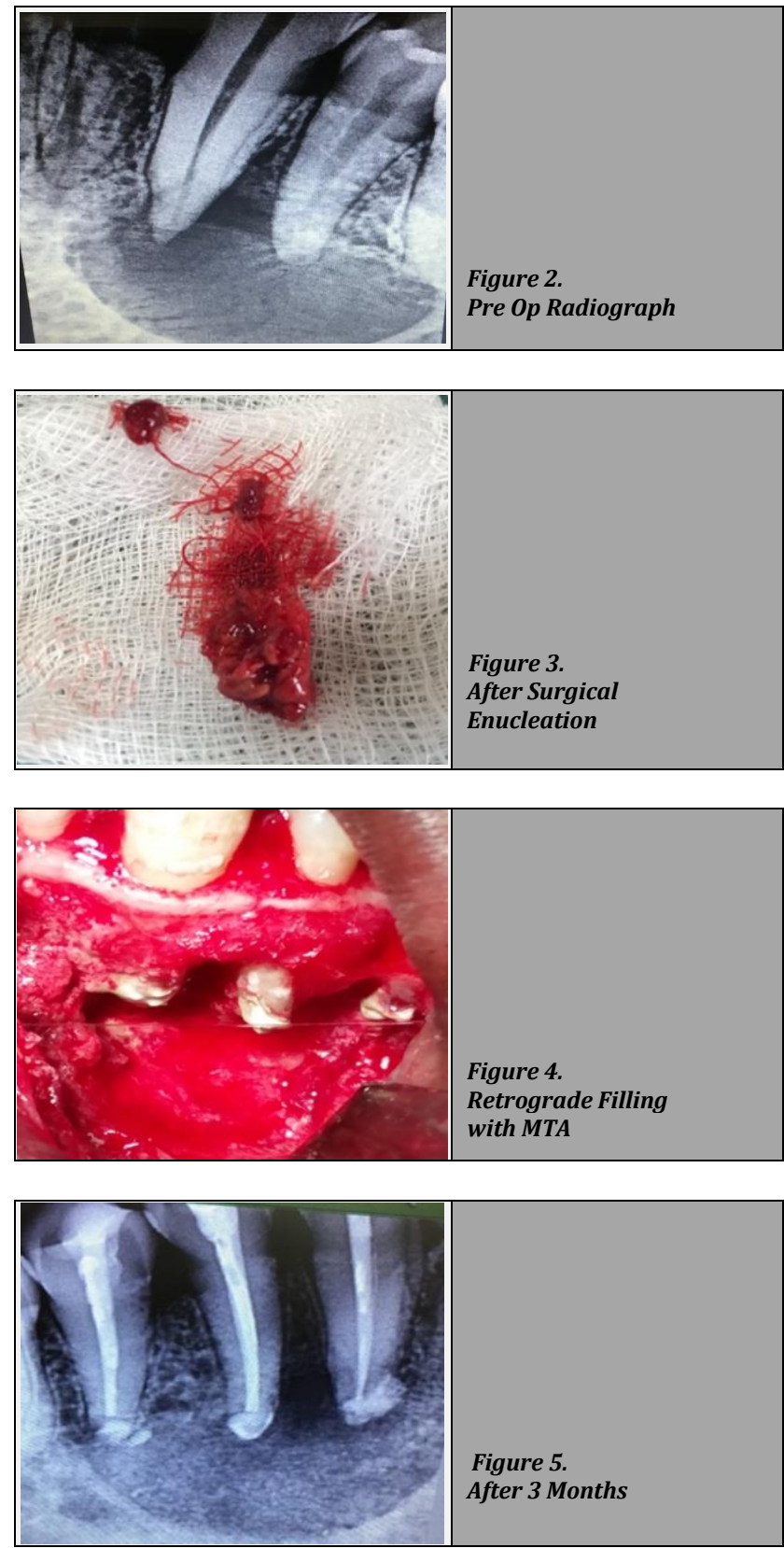

Figure 5. After 3 Months

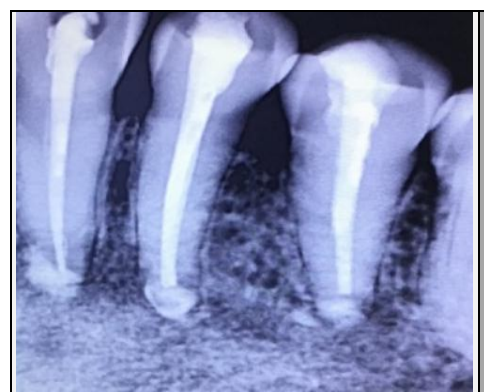

Figure 6.

After 24 Months 


\section{DISCUSSION}

Conventional or non-surgical root canal treatment is the first line of treatment for periapical endodontic lesions which have shown significant success rate. In cases where routine endodontic treatment fails even after meticulous cleaning, shaping and disinfection or in instances where root canal treatment is not possible, periapical surgery becomes necessary. After a surgical procedure, healing takes place either by repair or regeneration. ${ }^{5}$ The usage of a guided tissue regeneration membrane along with a suitable bone graft can result in the regeneration of the involved tissue along with functional rehabilitation. ${ }^{9}$ The decisive parameters for a successful healing after any surgical procedure is how efficiently the site is closed, ensuring blood supply to the surgical site, spatial relation and stability) ${ }^{10}$

In this case, platelet rich fibrin and bone graft was used for the treatment of intrabony defect. Platelet rich fibrin consists of a three dimensional structure of matrix of fibrin which has a lot of platelets and cytokines. Over a period of time as this three dimensional matrix disintegrates, the embedded cytokines and platelets are released over a period of seven to eleven days.

Platelet rich fibrin has abundant growth factors incorporated in it like the platelet derived growth factor, transforming growth factors, insulin like growth factor, epidermal growth factor, vascular epidermal growth factor, and fibroblast growth factor which are believed to promote new cell growth and differentiation. ${ }^{11-14} \mathrm{PRF}$ also controls the localised reaction manifested in the form of pain or swelling. 2,12

According to Simonpieri et al.,15 the usage of platelet rich fibrin during such surgical procedures has many advantages like the fibrin clot acts as a mechanical and biological connecting link between the bone particles. The incorporation of this fibrin network into the surgical site aids in cellular migration, particularly of the endothelial cells which are necessary for the neoangiogenesis, vascularization and survival of the graft. After that the platelet cytokines (PDGF, TGF- $\alpha$, IGF-1) are gradually released as the fibrin matrix is resorbed, thus creating a conducive process of healing. Finally, the presence of leukocytes and cytokines in the fibrin network plays a significant role in the self-regulation of the inflammation within the grafted material.

Preparation of platelet rich fibrin is very simplified, quick, and hassle free when compared to platelet-rich plasma (PRP) where artificial biochemical modification is required. Preparation of PRP requires the need to add an anticoagulant and subsequently neutralise it. Addition of a coagulation protein like thrombin is required to promote conversion of soluble fibrinogen to insoluble strands of fibrin in PRP. Whereas in contrast in PRF, these steps are not required also the biochemical handling of blood as well as the risks associated with the use of bovine-derived thrombin is considerably reduced. $4,5,7$ As the platelet rich fibrin is a derivative of one's own blood, chance that a cross contamination occurs is very low. Also, obtaining the platelet rich fibrin requires a minimal quantity of blood, which can be collected on the dental chair by a trained paramedic and avoids hospital admission or blood banks. ${ }^{16}$

Bone formation following periapical surgery can also be accelerated by placing bone graft into the bony defect.17,18 The ideal bone graft replacement material should be biologically inert, non-carcinogenic, easy to handle to fit into the osseous defect and structurally stable. It acts like a foundation for osteogenesis and should resorb at a slower pace so that the new bone formed can occupy its place ${ }^{19}$ In this case, bone graft material was used and a barrier membrane was used as there was not enough marginal bone.20-22 The histological examination of the enucleated lesion revealed periapical granuloma. The histopathologic evaluation of the granulation tissue is important to rule out any potential malignancies associated with periradicular lesions. ${ }^{23}$

\section{CONCLUSIONS}

Periradicular surgery performed with PRF and bone graft has exhibited good results in the healing of a large periapical lesion.

Ethical clearance for this case was obtained from the institutional ethical committee of Saveetha Dental College, Chennai.

Financial or Other Competing Interests: None.

\section{REFERENCES}

[1] Mazumbar P, Bhunia S. Treatment of periapical lesion with platelet rich fibrin. Indian Med Gazette 2013:28-33.

[2] Singh S, Singh A, Singh S, et al. Application of PRF in surgical management of periapical lesions. Natl J Maxillofac Surg 2013;4(1):94-9.

[3] Mazumdar S, Joshi S, Ansari S. Experiences with the use of PRF (Plasma Rich Fibrin) in enucleated cystic cavity. J Indian Dent Assoc 2014;8(6):19-26.

[4] Hiremath H, Motiwala T, Jain P, et al. Use of secondgeneration platelet concentrate (platelet-rich fibrin) and hydroxyapatite in the management of large periapical inflammatory lesion: a computed tomography scan analysis. Indian J Dent Res 2014;25(4):517-20.

[5] Shivashankar VY, Johns DA, Vidyanath S, et al. Combination of platelet rich fibrin, hydroxyapatite and PRF membrane in the management of large inflammatory periapical lesion. J Conserv Dent 2013;16(3):261-4.

[6] Shivashankar VY, Johns DA, Vidyanath S, et al. Platelet rich fibrin in the revitalization of tooth with necrotic pulp and open apex. J Conserv Dent 2012;15(4):395-8.

[7] Raja VS, Naidu EM. Platelet-rich fibrin: evolution of a second generation platelet concentrate. Indian J Dent Res 2008;19(1):42-6.

[8] Kiran NK, Mukunda KS, Tilak Raj TN. Platelet concentrates: a promising innovation in dentistry. J Dent Sci Res 2011;2:50-61.

[9] Bhandari S, Ashwini TS, Naik R, et al. Regenerative periapical surgery: a case report. Dent Hypotheses 2013;4(2):61-6

[10] Boyapati L, Wang HL. The role of stress in periodontal disease and wound healing. Periodontol 2000 2007;44:195-210. 
[11] Gassling V, Douglas T, Warnke PH, et al. Platelet-rich fibrin membranes as scaffolds for periosteal tissue engineering. Clin Oral Implants Res 2010;21(5):543-9.

[12] Corso MD, Toffler M, Ehrenfest DM. Use of autologous leucocyte and platelet rich fibrin (L-PRF) in post-avulsion sites: an overview of Choukran's PRF. J Implant Adv Clin Dent 2010;1:27-35.

[13] Deenadayalan E, Kumar A, Tewari RK, et al. Management of large periapical lesion with the combination of second generation platelet extract and hydroxyapatite bone graft: a report of three cases. J Clin Diagn Res 2015;9(1):D24-7.

[14] Nagaveni NB, Kumari KN, Poornima P, et al. Management of an endo-perio lesion in an immature tooth using autologous platelet-rich fibrin: a case report. J Indian Soc Pedod Prev Dent 2015;33(1):69-73.

[15] Simonpieri A, Del Corso M, Sammartino G, et al. The relevance of Choukroun's platelet-rich fibrin and metronidazole during complex maxillary rehabilitations using bone allograft. Part II: implant surgery, prosthodontics, and survival. Implant Dent 2009;18(3):220-9.

[16] Naik B, Karunakar P, Jayadev M, et al. Role of platelet rich fibrin in wound healing: a critical review. J Conserv Dent 2013;16(4):284-93.
[17] Saad AY, Abdellatief EM. Healing assessment of osseous defects of periapical lesions associated with failed endodontically treated teeth with use of freeze-dried bone allograft. Oral Surg Oral Med Oral Pathol 1991;71(5):612-7.

[18] Lalabonova H, Daskalov H. Jaw cysts and guided bone regeneration (a late complication after enucleation). J Int Med Assoc Bulg Annu Proc (Sci Pap) 2013;19(4):401-3.

[19] Jansson L, Ehnevid H, Lindskog S, et al. Development of periapical lesions. Swed Dent J 1993;17:85-93.

[20] Sreedevi P, Varghese N, Varugheese JM. Prognosis of periapical surgery using bonegrafts: a clinical study. J Conserv Dent 2011;14(1):68-72.

[21] von Arx T, Alsaeed M. The use of regenerative techniques in apical surgery: a literature review. Saudi Dent J 2011;23(3):113-27.

[22] Douthitt JC, Gutmann JL, Witherspoon DE. Histologic assessment of healing after the use of a bioresorbable membrane in the management of buccal bone loss concomitant with periradicular surgery. J Endod 2001;27(6):404-10.

[23] Philipsen HP, Srisuwan T, Reichart PA. Adenomatoid odontogenic tumor mimicking a periapical (radicular) cyst: a case report. Oral Surg Oral Med Oral Pathol Oral Radiol Endod 2002;94:246-8. 